\title{
Efficiency of Plating Media and Enrichment Broths for Isolating Salmonella Species from Human Stool Samples: A Comparison Study
}

\author{
Apichai Srijan, Boonchai Wongstitwilairoong, Ladaporn Bodhidatta, Carl Mason \\ Department of Enteric Diseases, Armed Forces Research Institute of Medical Sciences, US Army Medical \\ Component, Bangkok, Thailand \\ Email: apichais.fsn@afrims.org
}

Received 4 September 2015; accepted 7 December 2015; published 10 December 2015

Copyright (C) 2015 by authors and Scientific Research Publishing Inc.

This work is licensed under the Creative Commons Attribution International License (CC BY). http://creativecommons.org/licenses/by/4.0/

(c) (i) Open Access

\section{Abstract}

A comparative study was performed to evaluate best practice culture media and enrichment broths for recovering Salmonella species from human stool samples. A total of 1297 human stools were collected and processed in this study. Evaluation of agar media was carried out by direct plating (DP), 1096 stool samples were inoculated on Modified Semisolid Rappaport-Vassiliadis (MSRV), Xylose-Lysine-Deoxycolate (XLD), MacConkey (MAC), and Hektoen Enteric (HE) agars. Evaluation of enrichment broths were carried out by enrichment all 1297 stool samples in Selenite broth (SB), Rappaport-Vassiliadis (RV) broth, and Buffered Peptone Water (BPW), followed by plating on MSRV, MAC, and HE agars. A total of 102 Salmonella-positive stools by DP, 85.3\% (87/102) were recovered utilizing MSRV while recovery from XLD, MAC, and HE agars were $34.3 \%(35 / 102), 34.3 \%(35 / 102)$, and $29.4 \%(30 / 102)$ respectively. A total $299 / 1297$ stools samples were Salmonella-positive on at least one plating medium after enrichment procedure were $77.3 \%(177 / 299)$ for SB, $86.0 \%(197 / 299)$ and $78.6 \%(180 / 299)$ for RV and BPW respectively. All Salmonella isolated in this study was nontyphi Salmonella. Presently, the data suggest that the use of MSRV over MAC, HE, and XLD agars for isolation nontyphi Salmonella species from human stools is more efficacious. Additionally, use of MSRV in combination with MAC and HE agars following enrichment in RV broth enhances recovery of nontyphi Salmonella species. However, RV broth is inhibitory to typhi Salmonella, thus use of MSRV medium in combination with MAC, HE or XLD agars in direct plating following enrichment in non-selective BPW is an alternate method for recovery of both typhi and nontyphi Salmonella species contaminated in human stool samples. 


\section{Keywords}

\section{Human Stool Specimens, Culture Media, Salmonella, Isolation, Enrichment}

\section{Introduction}

Food-borne salmonellosis continues to be an important public health problem both in developed and developing countries [1]-[3]. Gastroenteritis is the most frequent illness derived from this infection and the use of stool or rectal swab samples are the main specimens used in diagnostic clinical laboratories. However, the presence of saprophytic flora, especially other coliforms in stool samples, has traditionally made isolation of Salmonella spp. difficult. Consequently, there is no ideal method for diagnosis of Salmonella infection and the development of highly selective media continues to be critical importance for diagnostic and treatment purposes. Conventional media base identification relies on the production of $\mathrm{H}_{2} \mathrm{~S}$ and $\mathrm{pH}$ indicator to detect lactose fermentation while bile salts and dyes inhibit saprophytic flora. However, these media types are inadequate to differentiate Salmonella spp. from other members of the Enterobacteriaceae, such as Proteus spp. and Citrobacter spp. [4]. In decreasing order of selectivity, MacConkey agar (MAC), Hektoen Enteric (HE) agar, Salmonella-Shigella (SS) agar, and Xylose-LysineDesoxycolate (XLD) agar are some of the more commonly used plating media in clinical laboratories.

Recently, several agar media containing chromogenic substrates for Salmonella-specific enzymes have been developed, i.e. Rambach agar [5], Salmonella Detection and Identification Medium (SM-ID; bioM'erieux, France), and ABC medium (Lab M. Ltd., Bury, United Kingdom). However, chromogenic agar media are less sensitive, but have greater specificity when compared to conventional media [6] [7]. Analternative method implementing enriched semisolid motility medium for isolating Salmonella from stool samples has been previously investigated. Jones \& Handley used a semisolid medium containing cacotheline as a selective medium after initial culture of suspected material in a broth containing hydroquinone [8]. Ino \& Graber used passage through semisolid medium in a "U" tube to recover Salmonella from cultures contaminated with Pseudomonas aeruginosa [9]. Stuart and Pivnick used selective motility (SM) medium modified with selenite F broth to make a semisolid medium contained in motility tubes to isolate Salmonella spp. from stool specimens. Data showed that the SM technique was equal or superior to those standard routine procedures employed in clinical laboratories and primary isolation of Salmonella from SM enrichment were relatively free from competing floras. Disadvantages to using the SM medium were the media was not shelf stable, could not be autoclaved and the migration time of Salmonella through the medium were lengthy [10].

Harper and Shortidge modified Shigella-Salmonella medium to a semisolid agar contained in Craigie tube to isolate Salmonella from stool specimens and found that $90 \%$ of specimens from which Salmonella were isolated were positive. Over $40 \%$ of these specimens would have been reported negative if the method had not been in use [11]. Modified Semisolid Rappaport-Vassiliadis (MSRV) medium is a modification of Rappaport-Vassiliadis enrichment broth for detecting motile Salmonella spp. in food products and feces [12] [13]. The original study on MSRV medium revealed a semisolid could be used as a rapid and sensitive method for isolation of Salmonella spp. from food products following pre-enrichment or selective enrichment [14] [15]. The efficacy of MSRV medium is based on the ability of Salmonella spp. to migrate through the selective medium ahead of other competing motile bacteria, thus producing opaque halos of growth surrounding the inoculating area. MSRV can be used as a plating medium for isolating Salmonella spp. (other than S. Typhi and S. Paratyphi A) from stool specimens with high sensitivity and specificity relative to chromogenic and conventional agar media [16] [17].

In order to evaluate the performance of MSRV, XLD agar media, and enrichment broths (e.g., BPW and RV) with our standard method for Salmonella spp. detection (MAC and HE agars and SB enrichment broth), a comparative study was conducted by direct plating for the isolation of Salmonella spp. Additionally, samples were enriched in SB broth (routine standard medium), BPW and RV broths, and prior to plating.

\section{Materials and Methods}

\subsection{Specimens}

The study was conducted from June 2003 to December 2004. Stool samples were collected from several diarr- 
hea surveillance studies conducted by the Department of Enteric Diseases, Armed Forces Research Institute of Medical Sciences and processed for isolation of enteric bacteria pathogens.

\subsection{Transport and Culture Media}

Modified Cary-Blair transport medium (Approximate formula per liter, Sodium Thyoglycollate $1.5 \mathrm{~g}$, Disodium Phosphate 1.1 g, Sodium Chloride 5.0 g, Agar 1.6 g). MAC agar (Difco, MD,USA), HE agar (Difco, MD,USA), MSRV (E. Merck, Darmstadt, Germany), Xylose-Lysine-Desoxycolate (XLD) agar, Selenite broth (SB) (BD Difco, USA), BPW (E. Merck, Darmstadt, Germany), and RV (R10 Broth, BD Difco, USA) were prepared according to the manufacturer's instructions. Prepared media for isolation and identification was tested with ATCC strains as quality control to assure quality of the prepared media.

\subsection{Method}

A total of 1297 stool samples were collected from both adults and children and were inoculated in SB, RV, and BPW enrichment broths prior to plating on MAC, HE, and MSRV. 1096 of the 1297 stools samples were used to compare the number of Salmonella-positive stool samples from MSRV, XLD, MAC, and HE agars by direct plating only with no enrichment step prior to plating.

\subsection{Direct Plating Method}

Stool suspensions (approximately 1:10 dilution) were prepared from fecal and rectal swabs by agitation of swabs in $0.85 \%$ sodium chloride solution and a drop of each stool suspension was used to inoculate MAC, HE, and XLD media and then streaked for growth of discrete colonies with a sterile loop. Inoculate 3 drops of stool suspension in separate spots on the surface of MSRV medium. MAC, HE, and XLD plates were incubated aerobically at $37^{\circ} \mathrm{C}$, for 18 - 24 hours, while MSRV media was incubated aerobically in an upright position at $42^{\circ} \mathrm{C}$, for 20 - 24 hours.

\subsection{Enrichment}

Approximately $0.5 \mathrm{~mL}$ of each stool suspension was inoculated into SB and BPW enrichment broths and incubated aerobically at $37^{\circ} \mathrm{C}$ for $18-24$ hours while inoculated RV broth was incubated at $42^{\circ} \mathrm{C}$ for $18-24$ hours, followed by subculture onto MAC, HE, and MSRV media and incubated as described above. Following the incubation period, MAC, HE, and MSRV media were examined for growth.

\subsection{Identification}

Up to three colonies suspected to be Salmonella spp. from each MAC, HE, and XLD agar media were inoculated onto KliglerIron agar (KIA), Lysine decarboxylase, Mannitol and Tryptone broth. Halos produced by swarming growth on MSRV medium were subcultured onto HE agar and incubated as described above. Suspected Salmonella isolates were confirmed by slide agglutination tests with O antisera for Salmonella according to the manufacturer's instructions (Polyvalent $\mathrm{O}$ antisera and individual group A-group 67, Serotest, S \& A REAGENT LAB, Bangkok, Thailand).

\subsection{Statistical Analysis}

The McNemar test was used to assess efficacy of plating media and enrichment broths. A p-value of $<0.05$ denoted statistical significance.

\section{Results}

Of the 1096 stool samples processed by direct plating, a total of 102 stools were positive for Salmonella on at least one plating medium in which 87 (85.3\%), 35 (34.3\%), 30 (29.4\%), and 35 (34.3\%) were isolated from MSRV, MAC, HE, and XLD, respectively (Figure 1). A total 299/1297 stools samples were Salmonella-positive on at least one plating medium after enrichment procedure (Figure 2). The number of samples containing Salmonella spp. using RV, BPW, and SB Enrichment broths were 197 (86.0\%), 180 (78.6\%), and 177 
(77.3\%), respectively. All Salmonella isolates were nontyphi Salmonella.

Direct plating on MSRV yielded significantly more Salmonella isolates when compared to MAC, HE, and XLD (p-value $<0.0001$ ) (Table 1). There was no statistical significance in numbers of Salmonella-positive stools when comparing MAC, HE and XLD. Enrichment with RV yielded significantly more Salmonella isolates when compared to BPW (p-value $=0.0405)$ and SB (p-value $=0.0126)$ (Table 1$)$. Use of MSRV in combination with MAC and HE when compared to MAC + HE increased recoverability of Salmonella isolates by 3.9\%, 55.5\%, and 30.6\% after enrichment cultures with RV, BPW, and SB respectively (Table 2).

\section{Discussion}

Improving isolation of Salmonella from human stool samples by using MSRV medium in clinical laboratories

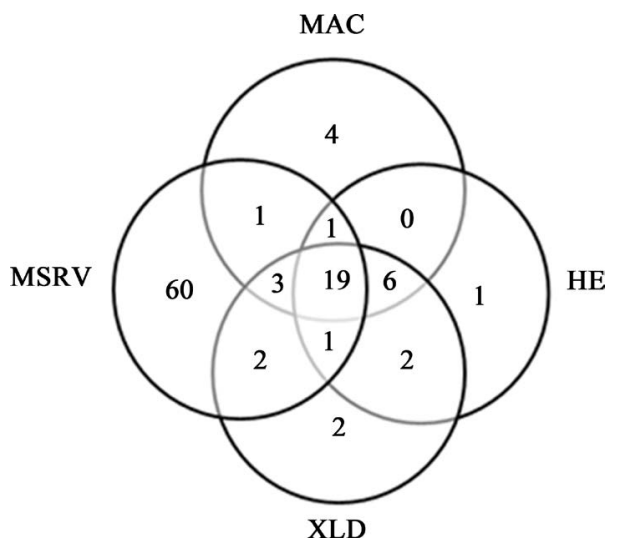

Figure 1. Venn diagram showing numbers of samples containing Salmonella on MAC, HE, XLD, and MSRV.

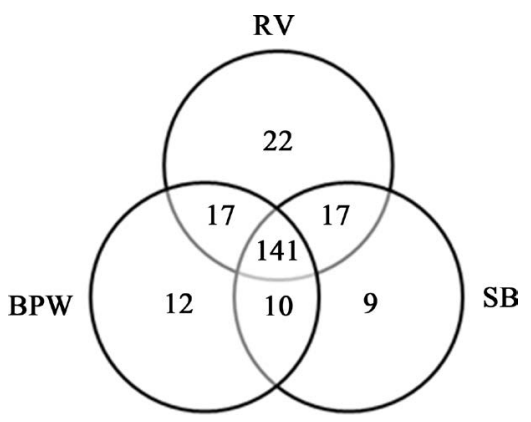

Figure 2. Venn diagram showing numbers of samples containing Salmonella recovered from RV, BPW, and SB enrichment broths.

Table 1. Statistical difference, based on numbers of Salmonella-positive stools, compared between four plating media and three enrichment broths.

\begin{tabular}{cc}
\hline Compared Media & Statistical Difference \\
\hline MAC vs. HE & p-value \\
XLD vs. MAC & 0.3877 \\
MSRV vs. MAC & 1.0000 \\
MSRV vs. HE & $<0.0001$ \\
MSRV vs. XLD & $<0.0001$ \\
XLD vs. HE & $<0.0001$ \\
RV vs. SB & 0.1797 \\
RV vs. BPW & 0.0126 \\
SB vs. BPW & 0.0405 \\
\hline
\end{tabular}


Table 2. Stool samples containing Salmonella compared among 4 selective agar media by direct plating and 3 enrichment broths.

\begin{tabular}{cccc}
\hline \multirow{2}{*}{ Enrichment broths } & Numbers (\%) of Salmonella-positive stool samples recovered from & \multirow{2}{*}{$\%$ Increase } \\
\cline { 2 - 3 } & MAC + HE & MAC + HE + MSRV & 3.9 \\
RV & $188(82.1)$ & $197(86.0)$ & 55.5 \\
BPW & $53(23.1)$ & $180(78.6)$ & 30.6 \\
\hline
\end{tabular}

has been described previously. Aspinal et al. (1992) reported a 21.3\% increase in isolates recovered by direct plating using MSRV compared to cultivable numbers utilizing selenite F broth enrichment prior to sub-culturing on brilliant green agar, while a $29 \%$ increase was indicated using Rappaport-Vassiliadis broth in conjunction with brilliant green agar. Furthermore, Dusch and Altwegg (1995) reported direct plating sensitivities of 63.4\% for MSRV in contrast to 34.1\% and 32.8\% for HE and Xylose-Lysine-Tergitol 4 (XLT4) respectively [17]. Ruiz et al. (1996) reported the number of Salmonella isolates recovered by direct plating on MSRV was also greater than those obtained on SS, Salmonella identification detection agar medium (SM-ID), Rambach agar, novobiocin-brilliant green-glycerol-lactose (NBGL) agar, and selenite-SS combination [6]. More recently, Ruiz et al. (1998) found a clear performance of MSRV over SS by direct plating with $24.7 \%$ greater sensitivity. They did not obtain significant differences when MSRV was compared to the selenite-SS combination, but the sensitivity of the selenite-MSRV combination was $8.2 \%$ greater than that of selenite-SS combination (p-value < 0.01) [18].

Presently, we intended to compare the yields of Salmonella from MSRV to MAC, HE and XLD only by DP method because it is known that number of Salmonella contained in stool samples to prior enrichment is much lower than after enrichment and is suitable for comparison. The performance of MSRV medium provided a 51.0\% increase in recoverability of non-typhoid Salmonella spp., when compared to MAC and XLD, while a 59.9\% increase when compared to HE agar. Although RV broth yielded the highest number of Salmonella-positive samples, there are several disadvantages of the medium as it is not suitable for detection of non-motile strains of Salmonella, S. Typhi and S. Paratyphi A [16] [19]. The yield obtained from BPW was highest when MSRV was used in conjunction with MAC and HE. In order to isolate all Salmonella serotypes, BPW should be used for enrichment followed by using MSRV in combination with MAC, HE. An additional advantage of BPW is that it enhances the growth of other enteric bacteria pathogens to include Shigella, Aeromonas, Plesiomonas shigelloides, and the diarrheagenic Escherichia coli. For these reasons, our laboratory has instituted using MSRV medium in direct plating and in combination with our standard media after enrichment culture in BPW for the improvement of isolating Salmonella spp. from human stool samples.

\section{Acknowledgements}

We thank Dr. Brett E. Swierczewski and Dr. Trent J. Peacock for reviewing the manuscript.

\section{References}

[1] Voetsch, A.C., Van Gilder, T.J., Angulo, F.J., et al. (2004) Foodnet Estimate of the Burden of Illness Caused by Nontyphoidal Salmonella Infections in the United States. Clinical Infectious Diseases, 38, 127-134. http://dx.doi.org/10.1086/381578

[2] Varavithaya, W., Vathanophas, K., Bodhidatta, L., et al. (1990) Importance of Salmonellae and Campylobacter jejuni in the Etiology of Diarrheal Disease Among Children Less than 5 Years of Age in a Community in Bangkok, Thailand. Journal of Clinical Microbiology, 2507-2510.

[3] Bodhidatta, L., Vithayasai, N., Eimpokalarp, B., Pitarangsi, C., Serichantalergs, O. and Isenbarger, D.W. (2002) Bacterial Enteric Pathogens in Children with Acute Dysentery in Thailand: Increasing Importance of Quinolone-Resistant Campylobacter. The Southeast Asian Journal of Tropical Medicine and Public Health, 33, 752-757.

[4] Devenish, J.A., Ciebin, B.W. and Brodsky, M.H. (1986) Novobiocin-Brilliant Green-Glucose Agar: New Medium for Isolation of Salmonellae. Applied and Environmental Microbiology, 52, 539-545.

[5] Rambach, A. (1990) New Plate Medium for Facilitated Differentiation of Salmonella spp. from Proteus spp. and Other Enteric Bacteria. Journal of Applied Environmental and Microbiology, 56, 301-303.

[6] Ruiz, J., Nunez, M.L., Lorente, I., Perez, J., Simarro, E. and Gomez, J. (1996) Performance of Six Culture Media for Isolation of Salmonella Species from Stool Samples. European Journal of Clinical Microbiology \& Infectious Diseases, 15, 922-926. http://dx.doi.org/10.1007/BF01690509 
[7] Nye, K.J., Fallon, D., Frodsham, D., et al. (2002) An Evaluation of the Performance of XLD, DCA, MLCB, and ABC Agars as Direct Plating Media for Isolation of Salmonella Enteric from Faeces. Journal of Clinical Pathology, 55, 286-288. http://dx.doi.org/10.1136/jcp.55.4.286

[8] Jones, R.E. and Handley, W.R.C. (1945) A Selective Medium for the Isolation of Salmonella Bacilli from Heavily Contaminated Material. Monthly Bulletin of the Ministry of Health and the Public Health Laboratory Service, 4, 107-111.

[9] Ino, J. and Graber, C.D. (1955) Recovery of Salmonella from Contaminated Cultures. US Armed Forces Medical Journal, 6, 586-587.

[10] Stuart, P.F. and Pivnick, H. (1965) Isolation of Salmonellae by Selective Motility Systems. Applied Microbiology, 13, 365-372.

[11] Harper, J. and Shortridge, K.F. (1969) A Selective Motility Medium for Routine Isolation of Salmonella. Journal of Hygiene (Lond), 67, 181-186. http://dx.doi.org/10.1017/S0022172400041589

[12] Rappaport, F., Konforti, N. and Navon, B. (1956) A New Enrichment Medium for Certain Salmonellae. Journal of Clinical Pathology, 9, 261-266. http://dx.doi.org/10.1136/jcp.9.3.261

[13] Vassiliadis, P., Trichopoulos, D., Pateraki, E. and Papaiconomou, N. (1978) Isolation of Salmonella from Minced Meat by the Use of a New Procedure of Enrichment. Zentralblatt fur Bakteriologie Originale B. Hygiene, Preventive Medezin, 166, 81-86.

[14] De Smedt, J.M., Bolderdijk, R.F., Rappold, H. and Lautenschlaeger, D. (1986) Rapid Salmonella Detection in Food by Motility Enrichment on a Modified Semi-Solid Rappaport-Vassiliadis Medium. Journal of Food Protection, 49, 510514.

[15] De Smedt, J.M. and Bolderdijk, R.F. (1987) Dynamics of Salmonella Isolation with Modified Semi-Solid Rappaport Vassiliadis Medium. Journal of Food Protection, 50, 658-661.

[16] Aspinall, S.T., Hindle, M.A. and Hutchinson, D.N. (1992) Improved Isolation of Salmonellae from Faeces Using a Semisolid Rappaport-Vassiliadis Medium. European Journal of Clinical Microbiology \& Infectious Diseases, 11, 936939. http://dx.doi.org/10.1007/BF01962379

[17] Dusch, H. and Altwegg, M. (1995) Evaluation of Five New Plating Media for Isolation of Salmonella Species. Journal of Clinical Microbiology, 33, 802-804.

[18] Ruiz, J., Lorente, I., Perez, J., Simarro, E. and Martinez, L. (1998) Evaluation of Methods for Isolation of Salmonella Species Using Modified Semisolid Rappaport-Vassiliadis Medium and Salmonella-Shigella Agar. European Journal of Clinical Microbiology \& Infectious Diseases, 17, 791-793. http://dx.doi.org/10.1007/s100960050188

[19] Goossens, H., Wauters, G., De Boeck, M., Janssens, M. and Butzler, J.P. (1984) Semisolid Selective-Motility Enrichment Medium for Isolation of Salmonellae from Fecal Specimens. Journal of Clinical Microbiology, 19, $940-941$. 